University of Nebraska - Lincoln

DigitalCommons@University of Nebraska - Lincoln

$2-11-2008$

\title{
Substrate-controlled growth and magnetism of nanosize Fe clusters on Pt
}

\author{
Ralph Skomski \\ University of Nebraska-Lincoln, rskomski2@unl.edu \\ J. Zhang \\ Max-Planck-Institut für Festkörperforschung \\ V. Sessi \\ Max-Planck-Institut für Festkörperforschung \\ Jan Honolka \\ Max-Planck-Institut für Festkörperforschung, honolka@fzu.cz \\ K. Kern \\ Max-Planck-Institut für Festkörperforschung \\ See next page for additional authors
}

Follow this and additional works at: https://digitalcommons.unl.edu/physicsskomski

Part of the Physics Commons

Skomski, Ralph; Zhang, J.; Sessi, V.; Honolka, Jan; Kern, K.; and Enders, Axel, "Substrate-controlled growth and magnetism of nanosize Fe clusters on Pt" (2008). Ralph Skomski Publications. 52.

https://digitalcommons.unl.edu/physicsskomski/52

This Article is brought to you for free and open access by the Research Papers in Physics and Astronomy at DigitalCommons@University of Nebraska - Lincoln. It has been accepted for inclusion in Ralph Skomski Publications by an authorized administrator of DigitalCommons@University of Nebraska - Lincoln. 


\section{Authors}

Ralph Skomski, J. Zhang, V. Sessi, Jan Honolka, K. Kern, and Axel Enders 


\title{
Substrate-controlled growth and magnetism of nanosize Fe clusters on Pt
}

\author{
R. Skomski \\ Department of Physics and Astronomy and Nebraska Center for Materials and Nanoscience, \\ University of Nebraska, Lincoln, Nebraska 68588, USA \\ J. Zhang, V. Sessi, J. Honolka, and K. Kern \\ Max-Planck-Institut für Festkörperforschung, Heisenbergstrasse 1, 70569 Stuttgart, Germany \\ A. Enders ${ }^{\text {a) }}$ \\ Department of Physics and Astronomy and Nebraska Center for Materials and Nanoscience, \\ University of Nebraska, Lincoln, Nebraska 68588, USA and Max-Planck-Institut für Festkörperforschung, \\ Heisenbergstrasse 1, 70569 Stuttgart, Germany
}

(Presented on 9 November 2007; received 10 September 2007; accepted 24 September 2007; published online 11 February 2008)

\begin{abstract}
The growth and magnetism of nanosize Fe clusters on Pt and other metal surfaces are investigated. Fe clusters have been fabricated directly on the substrates by buffer layer assisted growth under ultrahigh vacuum conditions. The mean cluster diameter and the average cluster spacing were controlled by the Fe coverage and the buffer layer thickness. The enhanced magnetic anisotropy of such clusters of diameters between 0.5 and $10 \mathrm{~nm}$ with respect to bulk is discussed. Interface anisotropy contributions are compared with direct dipolar cluster-cluster interaction and indirect interactions mediated by the substrate, including preasymptotic ferromagnetic interaction. It is found that this preasymptotic exchange is rather strong in exchange-enhanced substrates, such as $\mathrm{Pt}$, but it decreases rapidly with increasing distance between clusters and becomes negligible for the experimental cluster spacings in this work. Except for clusters that nearly touch each other, the leading interaction contributions are RKKY-type exchange and magnetostatic dipole interactions.
\end{abstract} (c) 2008 American Institute of Physics. [DOI: 10.1063/1.2828594]

\section{INTRODUCTION}

Clusters and dots of iron-series transition metals deposited on surfaces such as $\mathrm{Pt}$ and $\mathrm{Ag}$ are of potential interest for various applications, including ultrahigh-density magnetic recording. The advantage of $\mathrm{Pt}$ as substrate material is in its large spin-orbit coupling, which is a potential source of magnetic anisotropy for $3 d$ element adstructures. ${ }^{1-3}$ The focus of this work is on how the net interaction between the dots or clusters depends on dot size and separation. Strong exchange interactions are undesired in magnetic recording, because they may yield interaction domains and reduce the areal density. The interaction of magnetic moments through "simple" metals such as $\mathrm{Cu}$ is fairly well described as RudermanKittel-Kasuya-Yosida (RKKY)-type oscillations $J(d)$ $\sim \cos \left(2 k_{F} d\right) / d^{3}$, and it has been shown that these oscillations increase rather than decrease with increasing cluster size. ${ }^{4}$ In strongly exchange-enhanced Pauli paramagnets, such as Pd and Pt, the RKKY oscillations are only one consideration. These magnets are close to the onset of ferromagnetism (Stoner criterion), and the corresponding exchange enhancement modifies the wave-vector dependent susceptibility $\chi(k)$, which is of the RKKY type for free electrons. ${ }^{5,6}$ One consequence is a pronounced exponentially decaying preasymptotic contribution to the exchange coupling. ${ }^{7}$ The preasymptotic contribution is ferromagnetic and quite strong, although the RKKY oscillations continue to determine the interaction at long distances. At which cluster spacing does the preas-

\footnotetext{
${ }^{a)}$ Electronic mail: axel@unl.edu.
}

ymptotic behavior become important, especially compared to the involved thermal and magnetostatic energies?

\section{FE CLUSTERS ON PT AND AG}

The clusters in this work have been produced by buffer layer assisted growth (BLAG) ${ }^{8,9}$ This method includes the adsorption of a noble gas buffer layer, such as $\mathrm{Xe}$, on the crystalline substrate surface at temperatures below $50 \mathrm{~K}$, the deposition of the cluster material on the Xe by molecular beam epitaxy (MBE), and, finally, the desorption of the Xe layer by raising the substrate temperature above $100 \mathrm{~K}$. The clusters form directly on the Xe layer due to high mobility of the atomic species and low surface energy of Xe which prevents wetting, and grow in size during Xe desorption.

The cluster size and areal density are controlled during BLAG by two parameters, which are the thickness of the Xe buffer layer and the coverage of the cluster material. Examples of Fe clusters formed by BLAG on a crystalline $\mathrm{Pt}$ and $\mathrm{Ag}$ substrates are shown in the scanning tunneling microscopy (STM) images in Fig. 1. The smallest clusters, of less than $2 \mathrm{~nm}$ diameter, can be formed with $0.05 \mathrm{ML}$ (monolayers) Fe and 2 ML Xe (a). For the images (b)-(d), 2 $\mathrm{ML} \mathrm{Fe}$ and buffer layers of varied thickness between 5 and $45 \mathrm{ML}$ have been used. Clearly, higher Xe layer thickness or higher $\mathrm{Fe}$ coverage results in larger clusters. Also, the cluster density can be controlled via both the Fe and Xe coverage, and as a tendency, we find that higher cluster density is achieved with thinner Xe layers and comparatively large $\mathrm{Fe}$ coverage [see Fig. 1(b)]. The clusters have flattened hemi- 

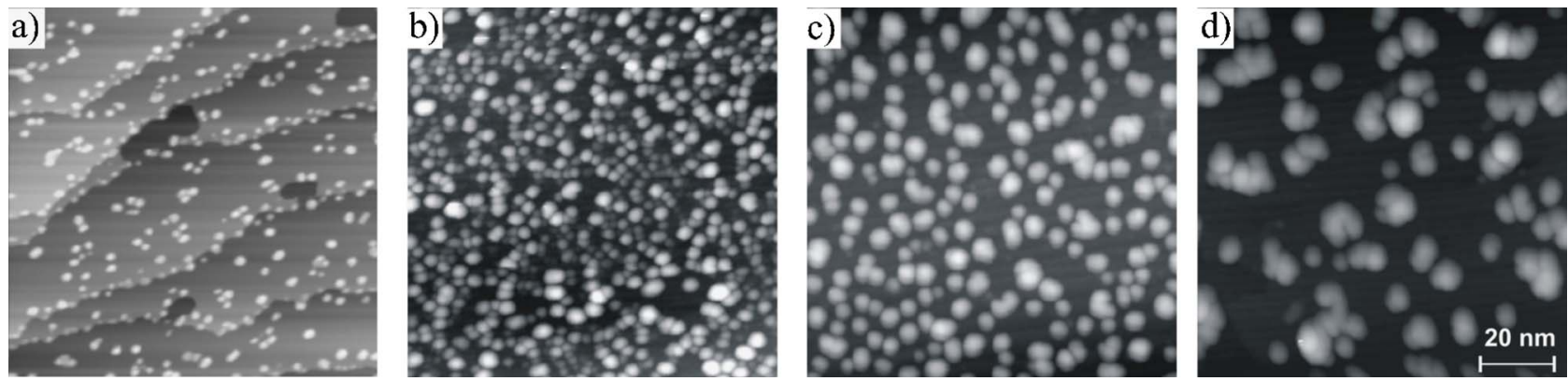

FIG. 1. (Color online) STM images of Fe clusters fabricated with buffer layer assisted growth. (a) $0.05 \mathrm{ML}$ Fe on Ag(111) using 2 ML Xe, [(b)-(d)] 2 ML Fe on Pt(997) using Xe buffer layers of 5, 18, and 45 ML thicknesses (Ref. 11).

spherical shape after making contact with the surface and their diameter increases from $1.5 \pm 0.7$ to $9.9 \pm 7.6 \mathrm{~nm}$, from (a) to (d). Since STM tends to overestimate the cluster size due to well-known tip convolution effects, the quoted cluster sizes are an upper limit.

For all shown clusters the magnetic anisotropy energy was found to be significantly enhanced with respect to bulk Fe. ${ }^{10,11}$ Similar experiments with Co clusters (formed of 0.05 ML Co with $5 \mathrm{ML} \mathrm{Xe)} \mathrm{even} \mathrm{showed} \mathrm{magnetic} \mathrm{anisotropy}$ values as high as $0.9 \mathrm{meV} / \mathrm{atom} .{ }^{12}$ Such high anisotropy is the result of the large Fe-Pt interface anisotropy caused by the hybridization of iron-series $3 d$ and heavy-transitionmetal $4 d / 5 d$ electrons. However, the question arises how and to what extent cluster-cluster interactions, mediated by the substrate, can contribute to the overall magnetic behavior.

\section{PREASYMPTOTIC COUPLING}

It is well known that conduction electrons in simple metals yield a RKKY interaction between magnetic impurities, ${ }^{5}$ and the same mechanism is realized between nanoscale units such as clusters. ${ }^{4,13}$ However, a different situation is encountered in exchange-enhanced Pauli paramagnets, such as $\mathrm{Pt}$ and Pd. These metals are close to the onset of ferromagnetism, which translates into an extra exchange contribution.

The starting point of the calculation is the wave-vector dependent susceptibility,

$$
\chi(k)=\frac{\chi_{\mathrm{o}}(k)}{1-U \chi_{\mathrm{o}}(k) / 2 \mu_{\mathrm{o}} \mu_{B}^{2}},
$$

where $\chi_{0}(k)$ is the noninteracting or Pauli susceptibility and the Stoner parameter $U$ (or $I$ ) describes the Coulomb interaction of the electrons. ${ }^{6}$ Note that Eq. (1) is essentially the Stoner criterion for the onset of ferromagnetism, and the Stoner-enhancement factor can also be written as $1 /(1$ $-D U)$, where $D$ is the density of states (DOS) at the Fermi level. The susceptibility $\chi_{\mathrm{o}}(k)$ describes the increase of the free electrons' kinetic energy due to inhomogenities of wave vector $k,{ }^{6,14}$

$$
\chi_{\mathrm{o}}(k)=\chi_{h}\left[1-k^{2} / 12 k_{F}^{2}+\mathrm{O}\left(k^{2}\right)\right] .
$$

The higher-order terms in this equation exhibit a complicated behavior with a singularity at $k=2 k_{F}$, which is the origin of the RKKY oscillations. ${ }^{5,6}$ Restricting the consideration to susceptibility terms quadratic in $k$ yields, by Fourier transformation, an exponential decay with a decay length $1 / \kappa$.
This is physically meaningful only if the quadratic term is very large, but analysis of Eq. (1) reveals that the Stonerenhancement factor $1 /(1-U D)$ enlarges both the range $1 / \kappa$ and the magnitude of the exponential term. The range of the preasymptotic decay scales as $1 /(1-U D)^{1 / 2}$. For $\mathrm{Cu}, \mathrm{Ag}, \mathrm{Pd}$, and $\mathrm{Pt}$, the theoretical values are 0.27, 0.43, 0.93, and $0.57 \AA$, respectively. The decay is also accessible by experiment, ${ }^{7}$ and experimental values are of order $2 \AA$ for Pd.

In the following, we neglect surface states, that is, electrons captured by and traveling along the surface, and approximate the geometry by the semi-infinite problem outlined in Fig. 2. Compared to infinite or bulk geometry, the volume of the spin-polarization cloud is halved, and the same is true for the net exchange interaction, because the interaction energy has the character of a volume integral over the space between the embedded particles.

The starting point of the calculation is the energy density, ${ }^{14,15}$

$$
\eta=\frac{1}{48 k_{F}^{2} D}(\nabla m)^{2}+\frac{1}{4}\left(\frac{1}{D}-U\right) m^{2},
$$

where $\mathrm{D}$ is the Pt or Pd density of states at the Fermi level and $U$ (or $I$ ) is the Stoner parameter. Minimization of Eq. (3) yields the Yukawa-type equation $\nabla^{2} m+\kappa^{2} m=0$, which has the well-known spherical solution $m_{s}(r)=c \exp (-\kappa r) / 4 \pi r$. Here, $r$ is the distance from the center of the cluster. Taking into account that the magnetization at the cluster surface is equal to that of iron, $m(R)=m_{\mathrm{o}}$, and that the moment of the Fe particle, $\mu_{\mathrm{o}},=4 \pi R^{3} m_{\mathrm{o}} / 3$, we obtain by integration over $4 \pi r^{2} \mathrm{~d} r$ the moment

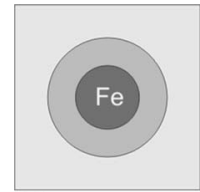

(a)

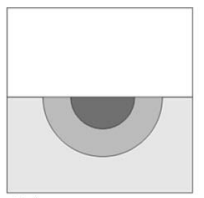

(b)

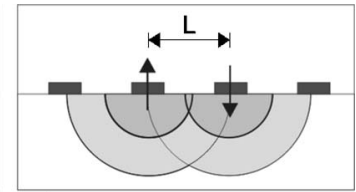

(c)
FIG. 2. Geometry of Fe particles embedded in Pt: (a) infinite (bulk) and (b) semi-infinite (surface). (c) Coupling between two clusters on a Pt surface (schematic). 


$$
\mu=3 \mu_{\mathrm{o}} \frac{1+\kappa R}{\kappa^{2} R^{2}}
$$

of the polarization cloud. Taking a single Co atom of moment $\mu_{0}=1.73 \mu_{B}$ and radius $R=1.24 \AA$, we obtain theoretical polarization-cloud predictions of $3.5 \mu_{B}$ for $\mathrm{Pt}$ and $7.3 \mu_{B}$ for Pt. Moments of this order of magnitude are indeed observed, for example, in dilute alloys. ${ }^{16} \mathrm{For} \mathrm{Cu}$, the corresponding moment is $1.4 \mu_{B}$, although $3 d$ atoms often loose their moment in simple metallic hosts, which is not taken into account by Eq. (4).

The exchange is obtained by setting $m_{ \pm}(\mathbf{r})$ $\sim m_{s}\left(\left|\mathbf{r}-\mathbf{R}_{1}\right|\right) \pm m_{s}\left(\left|\boldsymbol{r}-\mathbf{R}_{2}\right|\right)$ into Eq. (3) and evaluating the integral $E_{ \pm}=\int \eta\left(m_{ \pm}\right) \mathrm{d} V$. Here, the sign corresponds to ferromagnetic $(+)$ and antiferromagnetic $(-)$ exchange, and $J=$ $-\left(E_{+}-E_{-}\right)$. The calculation requires careful bookkeeping, including the boundary condition $m_{ \pm}(\mathbf{r})=m_{\mathrm{O}}$ at the clustermatrix interface, and yields, in lowest order,

$$
J=\frac{J_{\mathrm{o}}}{2} \frac{R^{2}}{R_{\mathrm{at}}^{2}} \exp (-\kappa L) .
$$

This equation, where $J_{\mathrm{o}}$ is an atomic exchange constant of the order of $J_{\mathrm{o}} / k_{B}=200 \mathrm{~K}$, is valid for large distances. Taking $R=1 \mathrm{~nm}$ and $L=3 \mathrm{~nm}$ yields an effective interaction of about $5 \mathrm{mK}$, corresponding to an interaction field of the order of 15 mikrotesla. By comparison, magnetostatic interaction fields between are typically of the order of a few millitesla.

\section{DISCUSSION AND CONCLUSIONS}

Equation (5) shows that the exchange increases quadratically with the radius $R$ of the clusters but is rapidly outweighed by the exponential term. This is different from the RKKY interaction between nanoparticles, ${ }^{4}$ which exhibits a power-law dependence on the particle or cluster separation. The oscillating RKKY tail dominates for well-separated particles, although $J$ may be positive or negative. For Pd thin films, the transition from exponential to power-law behavior occurs at about $10-12$ monolayers, ${ }^{7}$ and a similar transition is expected for the clusters considered here.

A striking feature of the interaction, Eq. (5), is its relative smallness. Compared to magnetostatic dipole interactions and RKKY interactions, it can safely be ignored for well-separated particles. In the limit of clusters that nearly touch each other, the functional dependence is different from Eq. (5), and the net effect is usually stronger, as analyzed for magnetic particles in a bulk matrix. ${ }^{17}$ Near-contact interactions are actually observed in some of our samples, where they improve the hysteresis-loop squareness. ${ }^{11}$ Note that the net RKKY interactions decay more rapidly than dipole interactions and are a small though not necessarily negligible correction for particles larger than about $1 \mathrm{~nm}^{4}$

In conclusion, we have investigated the exchange coupling through exchange-enhanced paramagnetic substrates. Progress in the fabrication of magnetic nanostructures makes it possible to deposit magnetic clusters with adjustable cluster sizes and separations, as shown in Fig. 1. Our results thus direct ways to control the magnetism of small clusters with the substrate. For cluster sizes and separations exceeding about $1 \mathrm{~nm}$, intercluster interactions have weak to moderate strengths. In decreasing order, these interactions are magnetostatic dipole interactions, RKKY interactions through the substrate, and exponentially decaying preasymptotic interactions through the substrate.

\section{ACKNOWLEDGMENTS}

This work has been supported by NSF-MRSEC and NCMN

${ }^{1}$ P. Gambardella, S. Rusponi, M. Veronese, S. S. Dhesi, C. Grazioli, A. Dallmeyer, I. Cabria, R. Zeller, P. H. Dederichs, K. Kern, C. Carbone, and H. Brune, Science 300, 1130 (2003).

${ }^{2}$ M. Komelj, D. Steiauf, and M. Fähnle, Phys. Rev. B 73, 134428 (2006). ${ }^{3}$ S. Bornemann, J. Minár, J. B. Staunton, J. Honolka, A. Enders, K. Kern, and H. Ebert, Eur. Phys. J. D 45, 529 (2007).

${ }^{4}$ R. Skomski, Europhys. Lett. 48, 455 (1999).

${ }^{5}$ N. W. Ashcroft and N. D. Mermin, Solid State Physics (Saunders, Philadelphia, 1976).

${ }^{6} \mathrm{P}$. Fulde, Electron Correlations in Molecules and Solids (Springer, Berlin, 1991)

${ }^{7}$ Z. Celinski and B. Heinrich, J. Magn. Magn. Mater. 99, L25 (1991).

${ }^{8}$ J. H. Weaver and G. D. Waddill, Science 251, 1444 (1991).

${ }^{9}$ J. H. Weaver and C. Haley, Surf. Sci. 518, 243 (2002).

${ }^{10}$ D. Repetto, J. Honolka, S. Rusponi, H. Brune, A. Enders, and K. Kern, Appl. Phys. A: Mater. Sci. Process. 82, 109 (2006).

${ }^{11}$ J. Zhang, D. Repetto, V. Sessi, J. Honolka, A. Enders, and K. Kern, Eur. Phys. J. D 45, 515 (2007).

${ }^{12}$ V. Sessi, J. Zhang, J. Honolka, K. Kuhnke, A. Enders, and K. Kern (unpublished).

${ }^{13}$ J. P. Pierce, M. A. Torija, Z. Gai, Junren Shi, T. C. Schulthess, G. A. Farnan, J. F. Wendelken, E. W. Plummers, and J. Shen, Phys. Rev. Lett. 92, 237201 (2004).

${ }^{14}$ Q.-N. Qi, R. Skomski, and J. M. D. Coey, J. Phys.: Condens. Matter 6, 3245 (1994).

${ }^{15} \mathrm{P}$. Mohn, Magnetism in the Solid State (Springer, Berlin, 2003).

${ }^{16}$ K.-H. Fischer and A. J. Hertz, Spin Glasses (Cambridge University Press, Cambridge, 1991).

${ }^{17}$ R. Skomski, A. Kashyap, Y. Qiang, and D. J. Sellmyer, J. Appl. Phys. 93, 6477 (2003). 